\title{
Role of ventriculoperitoneal shunting in patients with neoplasms of the central nervous system: An analysis of 59 cases
}

\author{
FARES NIGIM $^{1}$, JONATHAN F. CRITCHLOW ${ }^{2}$ and EKKEHARD M. KASPER ${ }^{1}$ \\ ${ }^{1}$ Division of Neurosurgery and ${ }^{2}$ Department of Surgery, Beth Israel Deaconess Medical Center, \\ Harvard Medical School, Boston, MA 02215, USA
}

Received June 4, 2015; Accepted August 12, 2015

DOI: $10.3892 / \mathrm{mco} .2015 .627$

\begin{abstract}
Approximately 1-5\% of patients with cerebral metastasis and $\sim 40 \%$ of patients with primary brain tumors suffer from hydrocephalus. These patients often exhibit a poor prognosis. The aim of the present study was to reassess the validity of ventriculoperitoneal shunting (VPS) with the assistance of the general surgeon in oncological patients. A total of 59 patients underwent first-time VPS at the Beth Israel Deaconess Medical Center (Boston, USA) between 2004 and 2012; 40 patients had hydrocephalus from brain metastasis and 19 from primary tumors. The analyzed independent variables included demographics, body mass index, past medical history, clinical presentation, indication for surgery, Karnofsky performance status (KPS) score and surgical technique; the dependent variables were postoperative symptoms and occurrence, cause and time of shunt failure. The outcomes were analyzed with the t-test and Kaplan-Meier estimates for shunt survival. The mean age of the patients was 57.2 years and the mean operative time was $50.4 \mathrm{~min}$. Symptomatic palliation was achieved in $93 \%$ of the cases; patients with severe symptoms, such as debilitating headaches, nausea and vomiting, benefited significantly from VPS. The mean follow-up time was 6.3 months; complications occurred in only 7 patients (11.8\%) during follow-up: 2 in the proximal shunt (1 infection and 1 obstruction), both requiring revision, 1 infection in the distal catheter requiring shunt removal, 2 cases of intracerebral bleeding that were monitored with computed tomography scans, 1 wound infection treated with antibiotics and 1 valve complication that required temporary revision. The initial and 3-month KPS scores were $65 \pm 16.4$ and $75 \pm 16.0$, respectively. The mean overall shunt survival
\end{abstract}

Correspondence to: Dr Ekkehard M. Kasper, Division of Neurosurgery, Beth Israel Deaconess Medical Center, Harvard Medical School, 110 Francis Street Suite 3B, Boston, MA 02215, USA

E-mail: ekasper@bidmc.harvard.edu

Key words: ventriculoperitoneal shunting, central nervous system neoplasms, brain metastasis, Karnofsky performance status, overall survival, leptomeningeal disease was 6.4 months (range, 1.0 day-76.0 months) from the placement of the VP shunt. At 3 months after VPS, 93.5\% of the patients remained alive with functioning shunts and at 1 year $87 \%$ of the shunts were still functioning. In conclusion, VPS remains a valid option for cancer patients with low KPS, as it improves the quality of life in such patients, even in the setting of previous infection, hemorrhage, or leptomeningeal disease, since shunt patency outlasts the overall survival of nearly all patients.

\section{Introduction}

The incidence of brain metastasis strongly depends on the primary tumor and, in certain types of cancer, also on the molecular subtype. Approximately 25-30\% of all cancer patients will develop brain metastasis during their life span (1). The incidence of brain metastasis per cancer type in adult patients has been reported to be as high as 45\% (range, 40-50\%) for patients with lung cancer, 20\% (range, 15-25\%) for breast cancer, $15 \%$ (range, $5-20 \%$ ) for malignant melanoma and $5 \%$ (range, 4-6\%) for patients with renal cell cancer; however, it is relatively rare in cancers of the gastrointestinal tract (2). The incidence of brain metastasis diagnosed intra vitam is increasing and the reasons for this are multifactorial: They include a factual increase in the incidence of cancers associated with tobacco use, such as lung cancer, but this incidence also increases secondarily to the prolonged survival of cancer patients with improving adjuvant therapy techniques. Finally, there is an increase in the detection rate due to the wider availability and more systematic utilization of magnetic resonance imaging (MRI) in upfront staging and during follow-up.

Irrespective of the existence of brain parenchymal metastasis, leptomeningeal seeding is considered to be a devastating complication of cancer and conveys a poor prognosis (3-7). The incidence of leptomeningeal metastases in patients with extracranial tumors is strictly correlated with the primary cancer type and is reportedly $\sim 30 \%$ (range, $22-64 \%$ ) in breast cancer, $16 \%$ (range, 10-26\%) in lung cancer, $11 \%$ (range, $7-15 \%$ ) in malignant melanoma, 6\% (range, 4-14\%) in gastrointestinal tract cancer and $1-7 \%$ in carcinomas of unknown primary origin (8-11). In addition to metastatic tumors, certain primary brain tumors may also be associated with leptomeningeal spread, with an incidence that varies widely from 10 to $32 \%$, and largely depends on tumor histology and patient age, 
but also varies with the respective referral pattern (8-11). Malignant leptomenigeal disease poses a unique challenge to the health care providers.

Approximately $1-5 \%$ of patients with solid brain tumors or leptomeningeal metastasis suffer from intracranial hypertension and/or hydrocephalus, with the incidence being even higher among patients with primary brain tumors $(6,12)$. Hydrocephalus may result from the obstruction of cerebrospinal fluid (CSF) pathways by any mass or dissemination of metastatic cells in the subarachnoid Virchow-Robin space, resulting in CSF malabsorption. The symptoms of hydrocephalus, such as headache, nausea, vomiting, gait disturbance, urinary incontinence, visual decline, cranial nerve palsy and even mental status changes, may be so disabling as to prevent systemic cancer treatment (6). These symptoms often do not respond well to conservative medical treatment with corticosteroids and analgesics or radiation. In fact, the majority of these patients reportedly present with comparably poor performance status and their condition in late-stage disease carries a very poor prognosis, with an overall survival (OS) (with treatment) of only 2-3 months in $\sim 50 \%$ of the patients, with only $10 \%$ surviving for 1 year $(3,13)$.

Ventriculoperitoneal shunting (VPS) may rapidly normalize intracranial pressure (ICP) in the setting of hydrocephalus $(6,14-18)$. However, despite the fact that it is considered a minor invasive procedure, it does involve certain risks, such as hemorrhage, shunt malfunction, or infection (19-21); in addition, it may rarely result in peritoneal carcinomatosis due to seeding from the central nervous system (CNS) tumors (22-39). Nevertheless, not all studies have encountered peritoneal seeding via VPS $(16,30,40)$.

There are several studies in the literature that have addressed radiation therapy, systemic chemotherapy and intrathecal chemotherapy specifically for the treatment for brain metastasis with/without leptomeningeal seeding (3,41-43). However, the number of published reports on the palliative surgical management of hydrocephalus secondary to brain metastasis and/or leptomeningeal seeding from malignant tumors is currently limited $(6,12,17,18)$.

The aim of this study was to reassess the validity, safety, and benefits of VPS as symptom-oriented palliative therapy for oncological patients with advanced-stage cancer. We also aimed to determine the risk/benefit ratio and investigate the outcomes of patients in whom previous medical management for hydrocephalic symptoms had failed and who were then selected for VPS.

\section{Patients and methods}

Patients. Data were prospectively collected from 59 consecutive adult patients diagnosed with hydrocephalus in the setting of brain metastasis or primary brain tumors, who underwent de novo VP shunt placement at the Beth Israel Deaconess Medical Center (BIDMC; Boston, USA) between April 2004 and July 2012. Patients who had previously undergone VPS and presented with shunt failure requiring shunt revision, as well as those with non-oncological indications for shunting, were excluded.

This retrospective cohort analysis was approved by our Institutional Review Board (no. 2011P-000101/4).
Medical record review. All pertinent medical records and related imaging studies were reviewed, including initial clinical and neurological presentation, primary tumor histology, Karnofsky performance status (KPS), recursive partitioning analysis (RPA) class status and radiographic imaging studies, such as pre- and postoperative computed tomography (CT)/MRI. Clinical symptom severity was assessed prior to and following VP placement. The durability (longevity) of the implanted VPS system was also examined via longitudinal follow-up appointments with clinical and imaging examinations, in order to detect possible occurrence, time and cause of shunt failure.

CNS metastasis and hydrocephalus. CNS metastasis was diagnosed based on available histopathology from systemic disease status and evaluation of cranial MRI findings. The presence of leptomenigeal metastasis at the time of VPS was diagnosed by the clinical status of the patient and the presence of characteristic leptomenigeal enhancing lesions on MRI, or by the presence of malignant cells found in a spinal tap sample of CSF. Hydrocephalus was classified as communicating hydrocephalus with leptomeningeal enhancement by MRI and high CSF protein content or positive CSF cytology, or as obstructive hydrocephalus due to a parenchymal or intraventricular mass.

Indications for VPS and procedure. The indications for placement of a VP shunt were: i) Clinical deterioration due to an increase in ICP despite aggressive medical ICP management, or ii) progressive ventriculomegaly in the setting of progressively developing neurological deficits.

All VP placements were performed under general anesthesia with endotracheal intubation. Preoperative MRI or CT imaging of the head was used for surgical planning. The proximal shunt catheter was passed into the lateral ventricle using a Ghajar guide or by the free-hand technique, depending on the surgeon's preference. Preset Medtronic Delta ${ }^{\circledR}$ valves (Medtronic, Dublin, Republic of Ireland), performance level 1.5, were used in all cases. The distal catheter was tunneled subcutaneously and its end was placed into the peritoneal cavity under direct visualization, either through a mini-laparotomy or via laparoscopic assistance. For intrathecal drug delivery and to provide access for CSF sampling, certain patients with suitable leptomeningeal disease (e.g., breast cancer) also had an Ommaya reservoir placed during the same operation via a separate incision. Postoperative CT scans of the head were performed on all patients to confirm the appropriate positioning of the proximal catheter and to rule out any procedural complications.

Follow-up. The most recent patient radiographic assessment (clinical visit or hospital discharge) was defined as the end-point of the follow-up period. For the purpose of this study and based on local referral patterns in our commonwealth, we hypothesized that patients who were not referred back to our hospital or office for any shunt-related problems, retained a functioning shunt for the entire study period. The end-points of the study were any occurrence of shunt revision, timing of shunt revision or shunt removal, and patient death. A Kaplan-Meier analysis was performed to evaluate the rate of shunt survival at 3 months and 1 year. 
Table I. Characteristics of the patient cohort.

\begin{tabular}{|c|c|}
\hline Characteristics & All shunts $(n=59)$ \\
\hline Mean age, years (median, range) & $57.2(61.9,22.0-81.6)$ \\
\hline \multicolumn{2}{|l|}{ Gender, no. (\%) } \\
\hline Male & $23(39.0)$ \\
\hline Female & $36(61.0)$ \\
\hline \multicolumn{2}{|l|}{ Indication for surgery, no. (\%) } \\
\hline Lung & $13(22.0)$ \\
\hline Breast & $11(18.0)$ \\
\hline Melanoma & $6(10.0)$ \\
\hline Renal & $3(5.0)$ \\
\hline Colorectal & $3(5.0)$ \\
\hline Ovarian & $2(4.0)$ \\
\hline Lymphoma & $2(4.0)$ \\
\hline CNS tumors & $19(32.0)$ \\
\hline \multicolumn{2}{|l|}{ Type of hydrocephalus, no. (\%) } \\
\hline Obstructive & $19(32.2)$ \\
\hline Communicating & $40(67.8)$ \\
\hline \multicolumn{2}{|l|}{ Presence of LMS } \\
\hline Yes & $20(34.0)$ \\
\hline No & $39(66.0)$ \\
\hline Mean Karnofsky score (range) & $65(30-100)$ \\
\hline \multicolumn{2}{|l|}{ RPA class, no. $(\%)$} \\
\hline I & $10(17.0)$ \\
\hline II & $16(27.0)$ \\
\hline III & $33(56.0)$ \\
\hline \multicolumn{2}{|c|}{ Progressive systemic disease, no. (\%) } \\
\hline Yes & $49(83.0)$ \\
\hline No & $10(17.0)$ \\
\hline
\end{tabular}

LMS, leptomeningeal seeding; RPA, recursive partitioning analysis; CNS, central nervous system.

\section{Results}

Patient characteristics. A total of 59 adult patients with hydrocephalus from CNS tumors or cranial metastasis underwent VPS at the BIDMC between 2005 and 2012. The mean age of the patients was 57.2 years (range, 22.0-81.6) years; our cohort included $23(39 \%)$ men and $36(61 \%)$ women. A total of 40 patients $(68 \%)$ had CNS metastasis from extracranial tumors, among those 13/59 (22\%) from lung cancer, 11/59 (18\%) from breast cancer, $6 / 59(10 \%)$ from melanoma, 3/59 (5\%) from renal cell cancer, $3 / 59$ (5\%) from colorectal cancer, 2/59 (4\%) from ovarian cancer and 2/59 (4\%) from lymphoma; of the entire cohort of 59 patients, $19(32 \%)$ patients had a primary CNS tumor.

A total of 19 (32.2\%) patients in this study group had obstructive hydrocephalus, whereas $40(67.8 \%)$ patients had communicating hydrocephalus; significant intracranial hypertension (ICP>20) was documented in $12 / 59$ patients $(20.3 \%)$ at the time of VPS. At the time of palliative VP shunt place-
Table II. Presenting symptoms and improvement following VP shunt placement.

\begin{tabular}{lcc}
\hline Presenting symptom & $\begin{array}{c}\text { No. of } \\
\text { patients (\%) }\end{array}$ & $\begin{array}{c}\text { Clinical } \\
\text { improvement } \\
\text { after VPS (\%) }\end{array}$ \\
\hline Headache, nausea, vomiting & $36(60.0)$ & $32(89.0)$ \\
Gait disturbance & $24(40.0)$ & $12(5.0)$ \\
Cognitive dysfunction & $18(30.0)$ & $8(440)$ \\
Seizure & $2(3.0)$ & $2(100.0)$ \\
Urinary incontinence & $1(2.0)$ & $1(1000)$ \\
\hline VP, ventriculoperitoneal. & & \\
\hline
\end{tabular}

Table III. Postoperative complications.

\begin{tabular}{lc}
\hline Complications & No. of patients $(\%)$ \\
\hline Overall postoperative complications & $7(11.8)$ \\
Shunt obstruction & \\
Proximal & 1 \\
Distal & 0 \\
Intracerebral bleeding & 2 \\
Infection & \\
Proximal shunt & 1 \\
Distal shunt & 1 \\
Wound & 1 \\
Valve revision & 1 \\
\hline
\end{tabular}

ment, 20/59 (33.8\%) of this patient cohort had leptomeningeal metastasis, whereas the remaining $39(66 \%)$ had parenchymal metastasis (Table I).

Symptoms. The majority of the patients presented with a variety of neurological symptoms, the most common being headache, nausea and vomiting $(60 \%)$, followed by gait disturbances $(40 \%)$, cognitive dysfunction such as memory impairment $(30 \%)$, seizures $(3 \%)$ and urinary incontinence $(2 \%)$ (Table II).

Preoperative assessment. The preoperative mean KPS score was found to be 65 (range, 30-100). The patients were also categorized according to the RPA class and the results revealed class I status in 10 (17\%), II in $16(27 \%)$, and III in $33(56 \%)$ patients. At the time of VP placement, there was documented progression of systemic disease in $49(83 \%)$ patients (Table I).

Outcome and complications. The vast majority of the patients $(55 / 59 ; 93.2 \%)$ from our cohort experienced an overall improvement in the neurological symptoms after surgery, but $4(6.8 \%)$ patients did not demonstrate a clear benefit from the procedure. The majority of the patients exhibited a significant clinical improvement within the first 3 or 4 days after surgery. 


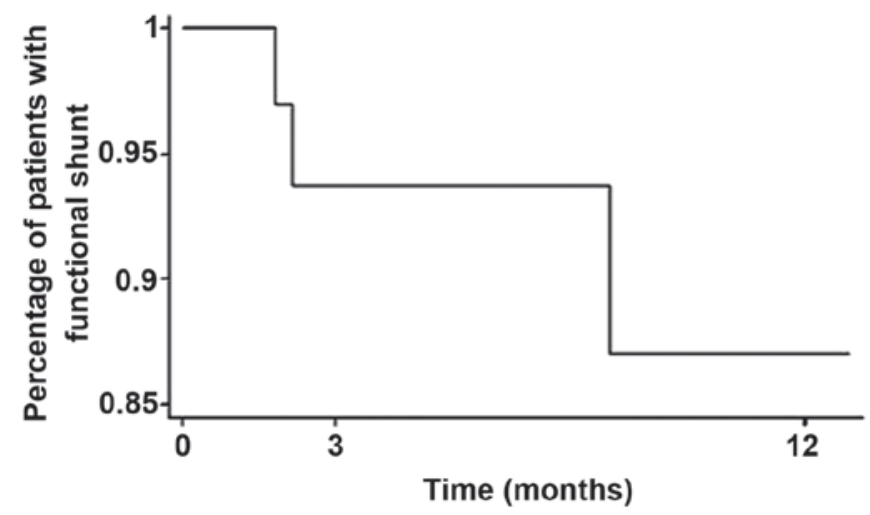

Figure 1. Mean overall survival without complications following ventriculoperitoneal (VP) shunt placement.

Improvement was most commonly observed in terms of the headache, nausea, vomiting and level of alertness. Cognitive function and gait disturbance did not respond as well to VPS, mainly reflecting the location of metastatic disease (Table II).

A total of $7(11.8 \%)$ postoperative complications were encountered (Table III). One patient developed proximal catheter obstruction and presented with changes in mental status requiring catheter replacement. Two patients suffered small intracranial hemorrhage from catheter placement, but were monitored by a series of CT scans and neither required shunt revision. One patient, who developed peritonitis from methicillin-resistant Staphylococcus aureus, was initially treated with antibiotics (vancomycin), but later underwent shunt removal. Another patient developed a proximal shunt infection (Staphylococcus aureus); this shunt was temporarily changed to an external ventricular drainage (EVD) prior to successful reinsertion, without any complications. Finally, 1 patient developed cellulitis at the abdominal incision, which was successfully treated with antibiotics alone, and 1 patient had a valve dysfunction that required revision. No further complications were encountered during the observation period (Table III).

Of note, there were no cases of clinically relevant intraperitoneal tumor dissemination via the inserted VP shunt, although the possibility of clinically silent intraperitoneal seeding cannot be excluded, as routine abdominal reimaging was not performed in all cases.

The mean overall shunt survival was 6.4 months (range, 1.0 day-76.0 months) from the placement of the VP shunt. At 3 months after VPS, $93.5 \%$ of patient were alive with functioning shunts and at 1 year $87 \%$ of the shunts remained functional (Fig. 1). We hypothesized that the shunts remained functional as long as the patients survived, and no deaths were recorded as a direct cosequence of early procedural or delayed shunt complications.

\section{Discussion}

Hydrocephalus in patients with cerebral metastases or primary brain tumors may severely compromise the patient's quality of life and poses a considerable challenge to the health care providers, since hydrocephalic CNS symptoms in terminally ill cancer patients are often medically uncontrollable. This leads to the difficult choice of either offering further surgical treatment, or transitioning the patient to pre-final hospice care. The available literature on this aspect of late-stage cancer patient care remains scarce. This issue is further complicated by the fact that a number of late- and end-stage brain cancer patients are in an immunocompromised state, due to leukopenia from myelosuppression following prior chemotherapy, or secondary to steroid treatment for edema control, which may lead to additional complications. Such frail patients are also at increased risk from anesthesia and may be at an increased risk of surgery- or shunt-related complications $(18,40,44,45)$.

The intracranial tumor burden in this setting may cause obstructive or malresorptive hydrocephalus, or lead to intracranial hypertension with altered CSF flow. Beyond general clinical symptoms that are amenable to medical treatment in this setting, intrathecal chemotherapy is not generally considered to be a suitable treatment option, since CSF compartmentalization prohibits effective drug delivery and tissue penetration and also increases the risk of neurotoxicity $(3,25,46,47)$. The experience with major surgical intervention in terms of any substantial benefit is limited in this setting, and quality of life for the remaining lifespan of such patients should be one of the goals of any health care provider.

In this study, we assessed a symptom-oriented approach and reported on the outcomes of 59 patients with primary extracranial or primary brain tumors who experienced clinical symptoms from their intracranial tumor burden causing hydrocephalus and/or intracranial hypertension. The patients analyzed in this cohort had been refractory to medical treatment of their symptoms despite aggressive management, and were therefore selected to undergo palliative VPS to restore CSF flow and treat intracranial hypertension.

In our cohort, the vast majority of the patients $(55 / 59$; 93.2\%) experienced immediate postoperative symptom relief. Global symptoms, such as headache, nausea and vomiting exhibited the most significant improvement following VPS, which compares favorably with the results from other studies on postoperative symptom relief in adult patients with metastatic CNS disease, which reportedly ranges between 70 and $80 \%(12,48,49)$.

Lee et al (18) reported their findings from a cohort of 50 patients with hydrocephalus from cerebral metastasis; in their experience, $80 \%$ of the patients improved after surgery, particularly with respect to preoperative headaches. Another study by Omuro et al (6) from the Memorial Sloan Kettering Cancer Center reported the outcome of 37 patients with leptomeningeal metastasis requiring VPS; in that particular cohort, 27/37 (77\%) patients exhibited some overall improvement following surgery, which translated into a substantial improvement in the quality of life of terminally ill patients. Several other studies and case reports in the literature have confirmed the efficacy of VPS as palliative therapy in patients with CNS metastasis or primary brain tumors (14-17).

In our cohort, the vast majority of the patients reported that headache, nausea and vomiting were the symptoms that improved the most following VPS, resolving in $89 \%$ of the cases; however, gait disturbances and cognitive dysfunction only improved in 50 and $40 \%$ of the patients, respectively, which may best be explained by the more local effects of the underlying disease. Of the 59 patients in our cohort, 
2 presented with delayed new-onset seizures, which may not be associated with the VPS procedure per se (since seizures are a well-known occurrence in patients with CNS tumors), whereas 1 patient presented with new-onset urinary incontinence 3 months after VPS, which may be explained as secondary to systemic disease progression, since his other preceding CNS symptoms improved following VP shunt placement. All these patients experienced improvement following surgery, without any recurrent CNS symptoms.

Of our 59 patients, 7 (11.8\%) experienced some form of postoperative complications, 4 of whom required shunt replacement with EVD prior to definitive care via shunt reinsertion; the remaining 3 cases were monitored only and did not require shunt revision or removal. The literature reports a rate of postoperative complications between 8 and $30 \%(6,12,48-50)$ in an unselected collective of VPS patients; therefore, our cohort is favorably at the lower end of that spectrum.

The mean OS of the patients in our cohort was 6.4 months, which is not necessarily a meaningful number, given the mixed underlying primary pathologies included in this study cohort; however, this number is in line with other studies $(3,5,7,12,17,1$ $8,43,47,51)$ and corroborates the observation that VPS in such patients is a durable form of therapy for symptom relief, particularly when high shunt patency rates are ensured. We did not observe any significant differences in post-VPS survival time according to primary malignancy type, which is in accordance with the existing literature $(12,18)$. This observation may be further explained by the fact that metastatic and, particularly, leptomeningeal CNS disease, is encountered at the very late stages of cancer, when the life expectancy of the patient is not associated with the therapy form assessed here, and remains very short. At 3 months after VPS placement, $93.5 \%$ of the shunts in our study group were found to be functioning, and at 1 year $87 \%$ of the shunts remained functional (Fig. 1).

Our results demonstrated that VPS in symptomatic metastatic patients frequently leads to marked improvement in hydrocephalic symptoms, even in the setting of leptomeningeal tumor spread. Based on our results and following a comprehensive review of the literature, we confirm the important role of VPS as palliation in end-stage patients suffering from either metastatic disease or primary brain tumors, with a poor prognosis. Based on our observations, these shunts show excellent durability that often exceeds the expected survival time and significantly improves the quality of the remaining lifetime, even when the overall prognosis is very poor.

In our cohort, we did not observe any clinical cases of peritoneal carcinomatosis secondary to seeding through the peritoneal catheter, although routine abdominal imaging was not performed. Another explanation may be that the patients had asymptomatic tumor deposits that remained clinically silent during the short OS period following VP shunt placement. Only an autopsy study may address this question in a definite way.

Further research should be focused on improving the diagnostic and therapeutic tools for patients with late-stage CNS malignancies, in order to identify patients who may benefit from this therapy earlier. More sensitive diagnostic tools are required to overcome the high false-negative rates of the currently available imaging modalities, which often fail to demonstrate leptomenigeal seeding (39), and the problem of negative cytology findings from CSF samples in this patient population. Beyond this, novel therapeutic modalities for targeted leptomenigeal disease are required, employing high-affinity agents that may be effectively applied despite CSF flow stagnation. Another useful tool would be to develop reliable, non-static, but rather dynamic VPS valve systems. The latter may allow for a timed 'switched-off' mode, thus enabling intermittent intrathecal chemotherapy, as well as long-term CSF shunting.

There were several limitations to this study. i) A major limitation lies with the fact that it is a retrospective study with a treatment paradigm that comes from a single institution and, thus, patient selection and their treatment pathways are subject to some selection bias; ii) another limitation of this study is the relatively small sample size; iii) long-term postoperative neurological evaluation was not performed in all patients, once the shunt was functional; and iv) not all patients were restaged to assess their clinical status regarding progression of systemic disease.

A prospective study with a larger sample size and more elaborate neurological scales to evaluate the postoperative condition over time for each patient, with accurate restaging for systemic disease, may add valuable information to this field of research.

In conclusion, although the prognosis of patients with CNS metastasis or malignant primary brain tumors in the clinical setting of hydrocephalus is very poor, VPS offers an effective, safe and valid palliative option for symptom relief and improvement of quality of life, even in patients with very poor overall prognosis. In addition to the improvement in neurological symptoms, VPS also allows for relatively simple and efficient delivery of intrathecal chemotherapy in those patients.

\section{References}

1. Kehrli P: Epidemiology of brain metastases. Neurochirurgie 45: 357-363, 1999 (In French).

2. Fidler IJ: The role of the organ microenvironment in brain metastasis. Semin Cancer Biol 21: 107-112, 2011.

3. Herrlinger U, Förschler H, Küker W, Meyermann R, Bamberg M, Dichgans J and Weller M: Leptomeningeal metastasis: Survival and prognostic factors in 155 patients. J Neurol Sci 223: 167-178, 2004.

4. Miralbell R, Tolnay M, Bieri S, Probst A, Sappino AP, Berchtold W, Pepper MS and Pizzolato G: Pediatric medulloblastoma: Prognostic value of p53, bcl-2, Mib-1 and microvessel density. J Neurooncol 45: 103-110, 1999.

5. Oechsle K, Lange-Brock V, Kruell A, Bokemeyer C and de Wit M: Prognostic factors and treatment options in patients with leptomeningeal metastases of different primary tumors: A retrospective analysis. J Cancer Res Clin Oncol 136: 1729-1735, 2010.

6. Omuro AM, Lallana EC, Bilsky MH and DeAngelis LM: Ventriculoperitoneal shunt in patients with leptomeningeal metastasis. Neurology 64: 1625-1627, 2005.

7. Taillibert S and Hildebrand J: Treatment of central nervous system metastases: Parenchymal, epidural and leptomeningeal. Curr Opin Oncol 18: 637-643, 2006.

8. Kaplan JG, DeSouza TG, Farkash A, Shafran B, Pack D, Rehman F, Fuks J and Portenoy R: Leptomeningeal metastases: Comparison of clinical features and laboratory data of solid tumors, lymphomas and leukemias. J Neurooncol 9: 225-229, 1990.

9. Pedersen PH, Rucklidge GJ, Mørk SJ, Terzis AJ, Engebraaten O, Lund-Johansen M, Backlund EO, Laerum OD and Bjerkvig R: Leptomeningeal tissue: A barrier against brain tumor cell invasion. J Natl Cancer Inst 86: 1593-1599, 1994.

10. Shapiro WR, Posner JB, Ushio Y, Chemik NL and Young DF: Treatment of meningeal neoplasms. Cancer Treat Rep 61: 733-743, 1977. 
11. Wasserstrom WR, Glass JP and Posner JB: Diagnosis and treatment of leptomeningeal metastases from solid tumors: Experience with 90 patients. Cancer 49: 759-772, 1982.

12. Chen CC, Kasper E and Warnke P: Palliative stereotactic-endoscopic third ventriculostomy for the treatment of obstructive hydrocephalus from cerebral metastasis. Surg Neurol Int 2: 76, 2011.

13. Glantz MJ, Jaeckle KA, Chamberlain MC, Phuphanich S, Recht L, Swinnen LJ, Maria B, LaFollette S, Schumann GB, Cole $\mathrm{BF}$, et al: A randomized controlled trial comparing intrathecal sustained-release cytarabine (DepoCyt) to intrathecal methotrexate in patients with neoplastic meningitis from solid tumors. Clin Cancer Res 5: 3394-3402, 1999.

14. Gonda DD, Kim TE, Warnke PC, Kasper EM, Carter BS and Chen CC: Ventriculoperitoneal shunting versus endoscopic third ventriculostomy in the treatment of patients with hydrocephalus related to metastasis. Surg Neurol Int 3: 97, 2012.

15. Schiff D, Kline C, Meltzer H and Auger J: Palliative ventriculoperitoneal shunt in a pediatric patient with recurrent metastatic medulloblastoma. J Palliat Med 12: 391-393, 2009.

16. Inamasu J, Nakamura Y, Saito R, Kuroshima Y, Mayanagi K, Orii $\mathrm{M}$ and Ichikizaki K: Postoperative communicating hydrocephalus in patients with supratentorial malignant glioma. Clin Neurol Neurosurg 106: 9-15, 2003.

17. Lokich J, Levine H and Nasser I: Malignancy-related hydrocephalus: Clinical features and results of ventricular peritoneal shunt procedure in three patients. Am J Clin Oncol 21: 366-368, 1998.

18. Lee SH, Kong DS, Seol HJ, Nam DH and Lee JI: Ventriculoperitoneal shunt for hydrocephalus caused by central nervous system metastasis. J Neurooncol 104: 545-551, 2011.

19. Handler MH and Callahan B: Laparoscopic placement of distal ventriculoperitoneal shunt catheters. J Neurosurg Pediatr 2: 282-285, 2008

20. Naftel RP, Argo JL, Shannon CN, Taylor TH, Tubbs RS, Clements RH and Harrigan MR: Laparoscopic versus open insertion of the peritoneal catheter in ventriculoperitoneal shunt placement: Review of 810 consecutive cases. J Neurosurg 115 151-158, 2011.

21. Roth J, Sagie B, Szold A and Elran H: Laparoscopic versus non-laparoscopic-assisted ventriculoperitoneal shunt placement in adults. A retrospective analysis. Surg Neurol 68: 177-184, 2007.

22. Brust JC, Moiel RH and Rosenberg RN: Glial tumor metastases through a ventriculo-pleural shunt. Resultant massive pleural effusion. Arch Neurol 18: 649-653, 1968.

23. Duffner PK and Cohen ME: Extraneural metastases in childhood brain tumors. Ann Neurol 10: 261-265, 1981.

24. Eralp Y,Saip P, Aydin Z,Berkman S and Topuz E: Leptomeningeal dissemination of ovarian carcinoma through a ventriculoperitoneal shunt. Gynecol Oncol 108: 248-250, 2008.

25. Glantz MJ, Cole BF, Recht L, Akerley W, Mills P, Saris S, Hochberg F, Calabresi P and Egorin MJ: High-dose intravenous methotrexate for patients with nonleukemic leptomeningeal cancer: Is intrathecal chemotherapy necessary? J Clin Oncol 16 : 1561-1567, 1998.

26. Hoffman HJ, Hendrick EB and Humphreys RP: Metastasis via ventriculoperitoneal shunt in patients with medulloblastoma. J Neurosurg 44: 562-566, 1976.

27. Hoffman HJ and Duffner PK: Extraneural metastases of central nervous system tumors. Cancer 56 (Suppl 7): 1778-1782, 1985.

28. Ingold B, Moschopulos M, Hutter G, Seeger H, Röthlisberger B, Landolt $\mathrm{H}$, Yonekawa $\mathrm{Y}$, Jochum $\mathrm{W}$ and Heppner FL: Abdominal seeding of an atypical teratoid/rhabdoid tumor of the pineal gland along a ventriculoperitoneal shunt catheter. Acta Neuropathol 111: 56-59, 2006.

29. Korones DN, Meyers SP, Rubio A, Torres C and Constine LSA: A 4-year-old girl with a ventriculoperitoneal shunt metastasis of a central nervous system atypical teratoid/rhabdoid tumor. Med Pediatr Oncol 32: 389-391, 1999.

30. Belongia $\mathrm{M}$ and Jogal S: Extraneural metastasis of a nongerminomatous germ cell tumor of the central nervous system in a pediatric patient with a ventriculoperitoneal shunt: A case report and review of the literature. J Pediatr Hematol Oncol 34: e12-e16, 2012.

31. Oberbauer RW, Tritthart H, Ascher PW, Walter GF and Becker H: Shunt metastases in posterior fossa tumors. Neuropadiatrie 10: 296-300, 1979
32. Paul MJ, Summers Y, Calvert AH, Rustin G, Brampton MH, Thatcher N and Middleton MR: Effect of temozolomide on central nervous system relapse in patients with advanced melanoma. Melanoma Res 12: 175-178, 2002.

33. Rickert CH: Extraneural metastases of paediatric brain tumours. Acta Neuropathol 105: 309-327, 2003.

34. Rubery ED and Wheeler TK: Metastases outside the central nervous system from a presumed pineal germinoma. Case report. J Neurosurg 53: 562-565, 1980.

35. Russell DS and Rubinstein LJ: Pathology of tumors of the nervous system. 4th edition. Arnold, London, pp206-207, 1977.

36. Sakata K, Yamada H, Sakai N, Hosono Y, Kawasako T and Sasaoka I: Extraneural metastasis of pineal tumor. Surg Neurol 3: 49-54, 1975.

37. Wakamatsu T, Matsuo T, Kawano S, Teramoto $\mathrm{S}$ and Matsumura H: Glioblastoma with extracranial metastasis through ventriculopleural shunt. Case report. J Neurosurg 34: 697-701, 1971.

38. Triolo PJ and Schulz EE: Metastatic germinoma (pinealoma) via a ventriculoperitoneal shunt. AJR Am J Roentgenol 135: 854-855, 1980

39. Han YP, Zhao Y, He XG and Ma J: Peritoneal metastasis of third ventricular atypical teratoid/rhabdoid tumor after VP shunt implantation for unexplained hydrocephalus. World J Pediatr 8: 367-370, 2012.

40. Berger MS, Baumeister B, Geyer JR, Milstein J, Kanev PM and LeRoux PD: The risks of metastases from shunting in children with primary central nervous system tumors. J Neurosurg 74: 872-877, 1991

41. Berg SL and Chamberlain MC: Systemic chemotherapy, intrathecal chemotherapy, and symptom management in the treatment of leptomeningeal metastasis. Curr Oncol Rep 5: 29-40, 2003.

42. Boogerd W, van den Bent MJ, Koehler PJ, Heimans JJ, van der Sande JJ, Aaronson NK, Hart AA, Benraadt J and Vecht ChJ: The relevance of intraventricular chemotherapy for leptomeningeal metastasis in breast cancer: A randomised study. Eur J Cancer 40: 2726-2733, 2004.

43. DeAngelis LM and Boutros D: Leptomeningeal metastasis. Cancer Invest 23: 145-154, 2005.

44. Lobotesis K, U-King-Im JM, Cross JJ, Gillard JH and Antoun NM: Gliomatosis peritonei associated with a ventriculo-peritoneal shunt. Clin Radiol 64: 95-99, 2009.

45. Zemack G and Romner B: Seven years of clinical experience with the programmable Codman Hakim valve: A retrospective study of 583 patients. J Neurosurg 92: 941-948, 2000.

46. Lassman AB, Abrey LE, Shah GD, Panageas KS, Begemann M, Malkin MG and Raizer JJ: Systemic high-dose intravenous methotrexate for central nervous system metastases. J Neurooncol 78: 255-260, 2006

47. Pentheroudakis G and Pavlidis N: Management of leptomeningeal malignancy. Expert Opin Pharmacother 6: 1115-1125, 2005.

48. Farahmand D, Hilmarsson $H$, Högfeldt $M$ and Tisell $M$ : Perioperative risk factors for short term shunt revisions in adult hydrocephalus patients. J Neurol Neurosurg Psychiatry 80: 1248-1253, 2009.

49. Hoh BL, Lang SS, Ortiz MV, Chi YY, Lewis SB and Pincus DW Lower incidence of reoperation with longer shunt survival with adult ventriculoperitoneal shunts placed for hemorrhage-related hydrocephalus. Neurosurgery 63: 70-74, 2008.

50. Reddy GK, Bollam P, Caldito G, Willis B, Guthikonda B and Nanda A: Ventriculoperitoneal shunt complications in hydrocephalus patients with intracranial tumors: An analysis of relevant risk factors. J Neurooncol 103: 333-342, 2011.

51. Lin N, Dunn IF, Glantz M, Allison DL, Jensen R, Johnson MD, Friedlander RM and Kesari S: Benefit of ventriculoperitoneal cerebrospinal fluid shunting and intrathecal chemotherapy in neoplastic meningitis: A retrospective, case-controlled study. J Neurosurg 115: 730-736, 2011. 